\title{
Increasing the horizontal resolution of ensemble forecasts at $\mathrm{CMC}$
}

\author{
G. Pellerin ${ }^{1}$, L. Lefaivre ${ }^{1}$, P. Houtekamer ${ }^{2}$, and C. Girard ${ }^{3}$ \\ ${ }^{1}$ Canadian Meteorological Centre, Montreal, Canada \\ ${ }^{2}$ Data Assimilation and Satellite Meteorological Division, Montreal, Canada \\ ${ }^{3}$ Recherche en Prevision Numerique Dorval, Canada
}

Received: 20 August 2002 - Revised: 21 March 2003 - Accepted: 22 April 2003

\begin{abstract}
Ensemble forecasts are run operationally since February 1998 at the Canadian Meteorological Centre, with outputs up to ten days. The ensemble size was increased from eight to sixteen members in August 1999. The method of producing the perturbed analyses consists of running independent assimilation cycles that use perturbed sets of observations and are driven by eight different models, mainly different in their physical parameterizations. Perturbed analyses are doubled by taking opposite pairs. A multi-model approach is then used to obtain the forecasts. The ensemble output has been used to generate several products. In view of increasing computing facilities, the ensemble prediction system horizontal resolution was increased to TL149 in June 2001. Heights at $500 \mathrm{hPa}$ and mean sea-level pressure maps are regularly used. Charts of precipitation with the probability of precipitation being above various thresholds are also produced at each run. The probabilistic forecast of the 24-h accumulated precipitation has shown skill as demonstrated by the relative operating characteristic (ROC). Verifications of the ensemble forecasts will be presented.
\end{abstract}

\section{Introduction}

The ensemble prediction system is running operationally at the Canadian Meteorological Centre (CMC) since February 1998, using 8 members of the SEF (Spectral Finite Element) model at resolution TL95 (Ritchie 1991). Adding the experimental runs, accomplished in development, the CMC ensemble prediction system has been running since January 1996. In August 1999, the ensemble size was doubled to 16 members. A different model, the Global Environment Multi-scale (GEM), was then used for the additional 8 members (see Côté et al., 1998). Section 2 will describe the method used for this multi-model approach. With the increasing computational facilities at CMC in 2001, it was decided to increase the horizontal resolution of the set. Sec-

Correspondence to: G. Pellerin (Gerard.pellerin@ec.gc.ca) tion 3 will describe the results obtained when the horizontal resolution of the SEF ensemble members increased from TL95 to TL149, and the resolution of the GEM ensemble members increased from 1.875 to 1.2 degrees. No fundamental changes to the methodology have been implemented since 1996, but as a result of these modifications, the scores have improved significantly. Section 3 will also describe the method used for verifications and the pertinence of the improvement as compared to previous year validations. Section 4 will cover a short discussion and outline the future work planned.

\section{Description of the ensemble prediction system}

The ensemble method used at CMC is described in Houtekamer et al. (1996) and Lefaivre et al. (1997). The basis of the method is to produce perturbed analyses through data assimilation procedures. In order to do so, 8 parallel analysis cycles are run quasi-independently of the deterministic operational analysis (TL199). In addition, the mean of the 8 analyses is calculated and then subtracted to that of the deterministic analysis. A fraction of this difference is then added to the original 8 perturbed analyses so that a total of 16 initial fields is obtained for the medium range forecasts (see Table 1). The characteristics of the perturbed cycles are summarised in Fig. 1.

First, each observation used to feed the analyses is perturbed. For observations with multiple levels, such as radiosondes, the vertical correlations are taken into account by performing an eigenvector analysis of the vertical covariance matrix. Random numbers, drawn from a Gaussian distribution with zero mean and unit variance, are then multiplied by eigenvectors times the root of the eigenvalues (e.g. Houtekamer, 1993). For single- level observation, the perturbations are simply independent random numbers with zero mean and the variance appropriate for that observation. The random values are different for each independent piece of information and are different from one perturbed cycle to the 
Table 1. Combination of modules for different model versions

\begin{tabular}{|c|c|c|c|c|c|c|c|}
\hline $\begin{array}{c}\text { SEF } \\
(\mathrm{T} 149)\end{array}$ & $\begin{array}{l}\text { Add ops } \\
\text { analysis }\end{array}$ & $\begin{array}{c}\text { Convection/ } \\
\text { Radiation }\end{array}$ & $\mathrm{GWD}^{0}$ & $\mathrm{GWD}^{0}$ version & Orography & $\begin{array}{l}\text { Number } \\
\text { of levels }\end{array}$ & $\begin{array}{l}\text { Time } \\
\text { level }\end{array}$ \\
\hline 1 & yes & Kuo/ Garand & strong & high altitude & $0.3 \sigma$ & 23 & 3 \\
\hline 2 & no & Manabe/ Sasamori & Strong & low altitude & $0.3 \sigma$ & 41 & 3 \\
\hline 3 & no & Kuo/ Garand & weak & low altitude & mean & 23 & 3 \\
\hline 4 & yes & Manabe/ Sasamori & weak & high altitude & mean & 41 & 3 \\
\hline 5 & yes & Manabe/ Sasamori & strong & low altitude & mean & 23 & 2 \\
\hline 6 & no & Kuo/ Garand & strong & high altitude & mean & 41 & 2 \\
\hline 7 & no & Manabe/ Sasamori & weak & high altitude & $0.3 \sigma$ & 23 & 2 \\
\hline 8 & yes & Kuo/ Garand & weak & low altitude & $0.3 \sigma$ & 41 & 2 \\
\hline control & mean & Kuo/ Garand & mean & low altitude & $0.15 \sigma$ & 41 & 3 \\
\hline $\begin{array}{l}\text { GEM } \\
\left(1.2^{\circ}\right)\end{array}$ & $\begin{array}{l}\text { Add ops } \\
\text { analysis }\end{array}$ & Deep convection & $\begin{array}{l}\text { Shallow } \\
\text { convection }\end{array}$ & Soil moisture & Sponge & $\begin{array}{l}\text { Number } \\
\text { of levels }\end{array}$ & Coriolis \\
\hline 9 & no & Kuosym $^{1}$ & new & $<20 \%$ & global & 28 & implicit \\
\hline 10 & yes & RAS $^{2}$ & old & $<20 \%$ & equatorial & 28 & implicit \\
\hline 11 & yes & RAS $^{2}$ & old & $<20 \%$ & global & 28 & implicit \\
\hline 12 & no & Kuosym $^{1}$ & old & $>20 \%$ & global & 28 & implicit \\
\hline 13 & no & Kuosym $^{1}$ & new & $>20 \%$ & global & 28 & explicit \\
\hline 14 & yes & Kuosym $^{1}$ & new & $<20 \%$ & global & 28 & implicit \\
\hline 15 & yes & Kuosym $^{1}$ & old & $<20 \%$ & global & 28 & implicit \\
\hline 16 & no & Kuosun ${ }^{3}$ & new & $>20 \%$ & global & 28 & explicit \\
\hline
\end{tabular}

${ }^{0}$ Gravity Wave drag (see McFarlane (1987) and McLandress and McFarlane (1993))

${ }^{1}$ Deep convection stream (see Wagneur (1991))

${ }^{2}$ Relaxed Arakawa-Schubert (see Moorthi and Suraez (1992))

${ }^{3}$ see Dastoor (1994)

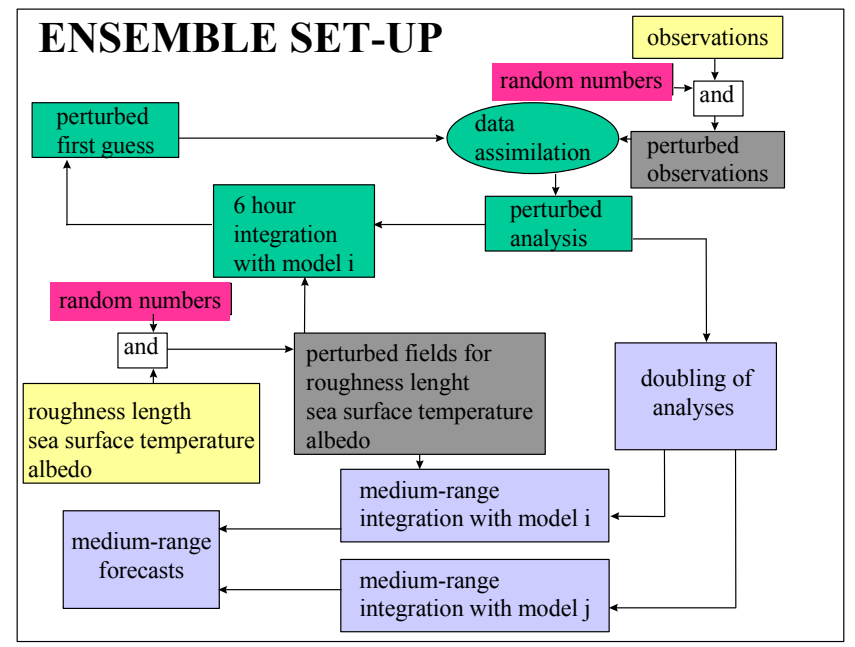

Fig. 1. Organisation chart of the ensemble set-up.

other. The analysis scheme used is the Optimal Interpolation (O/I) technique (Rutherford, 1972), which has the advantage of being efficient computerwise (the weights for the innovations are calculated once for the control, using a cholesky de- composition, and used for the 8 ensemble members as well).

Second, each of the models used in the assimilation cycles, although based on the same spectral model (Ritchie, 1991) with a horizontal resolution of TL149 and a horizontal diffusion in $\nabla^{4}$, have different switches activated. These different configurations, used to produce the trial fields of each cycle, are described in the top half of Table 1. In addition, some physical parameters are set with random values (horizontal diffusion, minimal roughness length over SST, time filter). Perturbations are also introduced in the surface forcing through modifications (gaussian perturbations) of the sea surface temperature, the albedo and the roughness length fields. The GEM models are only used for the forecasts.

Once a day, at 00:00 UTC, 10-day forecasts are produced using:

- 8 perturbed analyses, half of them obtained by adding a fraction of the difference between the ensemble mean and the operational analysis (see 2 nd column of Table 1), using the same model options as the ones used to produce the trial fields as detailed in top half of Table 1;

- the control run, obtained from an analysis with no perturbed observations and with intermediate model options (the deterministic model keys are mostly used); 
Validation of ensemble forecasts $(500 \mathrm{hPa})$ over $\mathrm{NH}$ DECEMBER 2000

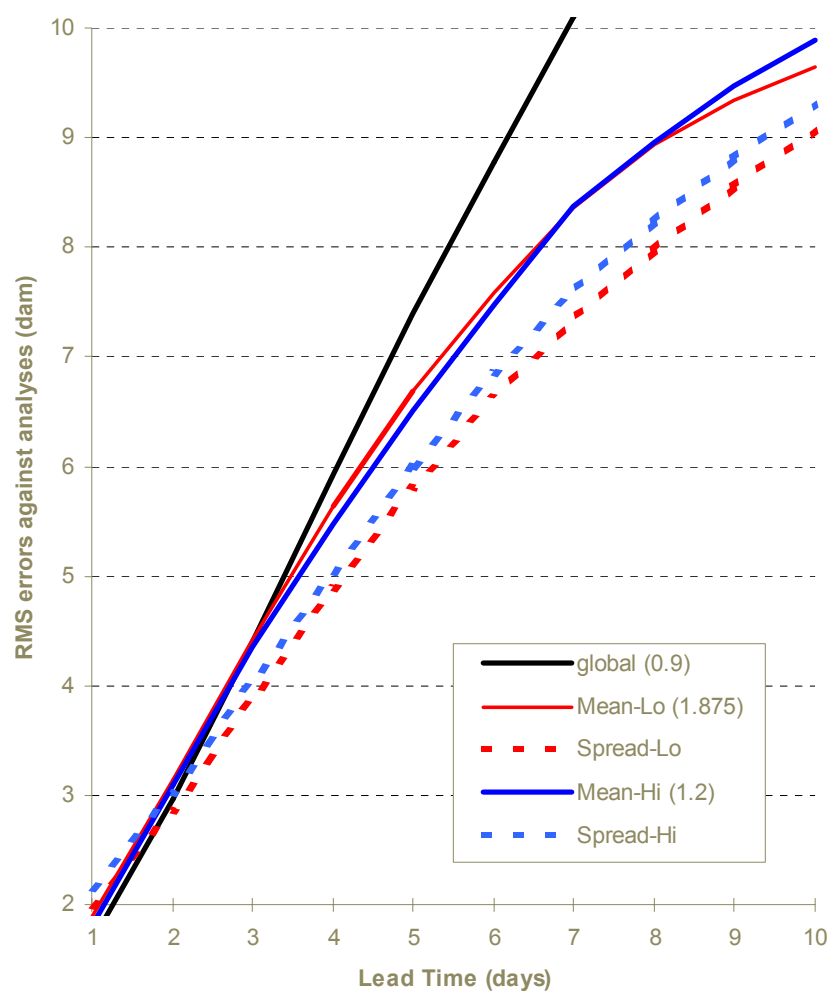

Fig. 2. Verification (root mean square error) of height at $500 \mathrm{hPa}$ over northern Hemisphere for the low resolution ensemble (solid red line) as compared to the high resolution ensemble (solid blue line) and the deterministic model (solid black line); spreads in the ensemble are dashed lines.

- 8 perturbed analyses, half of them obtained by adding a fraction of the difference between the ensemble mean and the operational analysis (see 2nd column of Table 1), using this time the GEM model with its own set of perturbations, detailed in bottom half of Table 1 .

\section{Verification results}

By December 2000, a parallel run was set-up with the higher resolution ensemble. It was evaluated over the winter season (December to February) and gave acceptable results. It was then decided to pursue testing for a few months to assess the gain in the precipitation scores. The next sections will describe verification scores for the RMS errors and Talagrand diagrams for the $500 \mathrm{hPa}$ height field and for precipitation forecasts. The ROC curves provide a good representation of the quality of probabilistic precipitation forecasts (Mason, 1982). A comparison of ROC curves calculated for both low and higher resolutions will give a good indication of the improvement of precipitation forecasts. This will be shown in the next sections.

\subsection{RMS errors for the $500 \mathrm{hPa}$ height field}

The December 2000 verification for the two ensemble sets is seen in Fig. 2, where a series of Root Mean Square (RMS) errors of height at $500 \mathrm{hPa}$ for Northern Hemisphere are shown. The solid line indicates the RMS error for the operational model (GEM $0.9^{\circ} \mathrm{L} 28$ ), while the thin blue and red lines, represent the ensemble mean RMS errors for the high and low resolution runs, respectively. The dotted lines represent the spread of the ensembles (blue for high resolution). One would like to see the RMS errors for the ensemble mean smaller than for the high resolution model and the spread of the ensemble close to the ensemble mean error. Although after 7 days, the scores of the high resolution ensemble are not as good as that of the low resolution one, the spread remains better for all forecast times. This was also the case for the month of July 2001 (not shown), the higher resolution ensemble had increased spread and reduced mean RMS errors throughout the 10 day period.

\subsection{Talagrand diagrams for the $500 \mathrm{hPa}$ height field}

To verify if the spread of an ensemble encompasses the verifying analysis, Talagrand et al. (1997) proposed a statistical method for displaying bias and dispersion within the ensemble. The so-called Talagrand diagrams are obtained by checking where the verifying analysis usually falls with respect to the ensemble forecast data (arranged in increasing order at each grid point). The first (last) bin is selected if the analysed value is lower (higher) than any of the values in the ensemble.

Since all perturbations are intended to represent equally likely scenarios, this distribution should be flat. Several common problems can be diagnosed from the diagrams:

- a U-shape diagram is obtained if the spread in the ensemble is typically too small;

- if the spread in the ensemble is too big one obtains an n-shape (highest in the middle);

- if the diagram is asymmetric the model has a bias to one side. An L-shape would correspond to a warm bias for the model.

Operationally, these diagrams are produced every month. For our comparison we show the low (TL95) and high resolution (TL149) diagrams for the $500 \mathrm{hPa}$ (global coverage) in Figs. 3 and 4 respectively. These diagrams are for all lead times of the month of February 2001. It is encouraging to see that the diagrams display nearly flat distributions with no marked systematic biases, except a slight warm bias which does not seem to have changed with the increase in resolution. It is true that there is too much spread at day 1 , especially for the high resolution ensemble. We think that this may be due to the perturbations of the observations which were set to high (factor 1.8) during the analysis cycles. 
Talagrand diagrams for $500 \mathrm{hPa}$, global area

February 2001

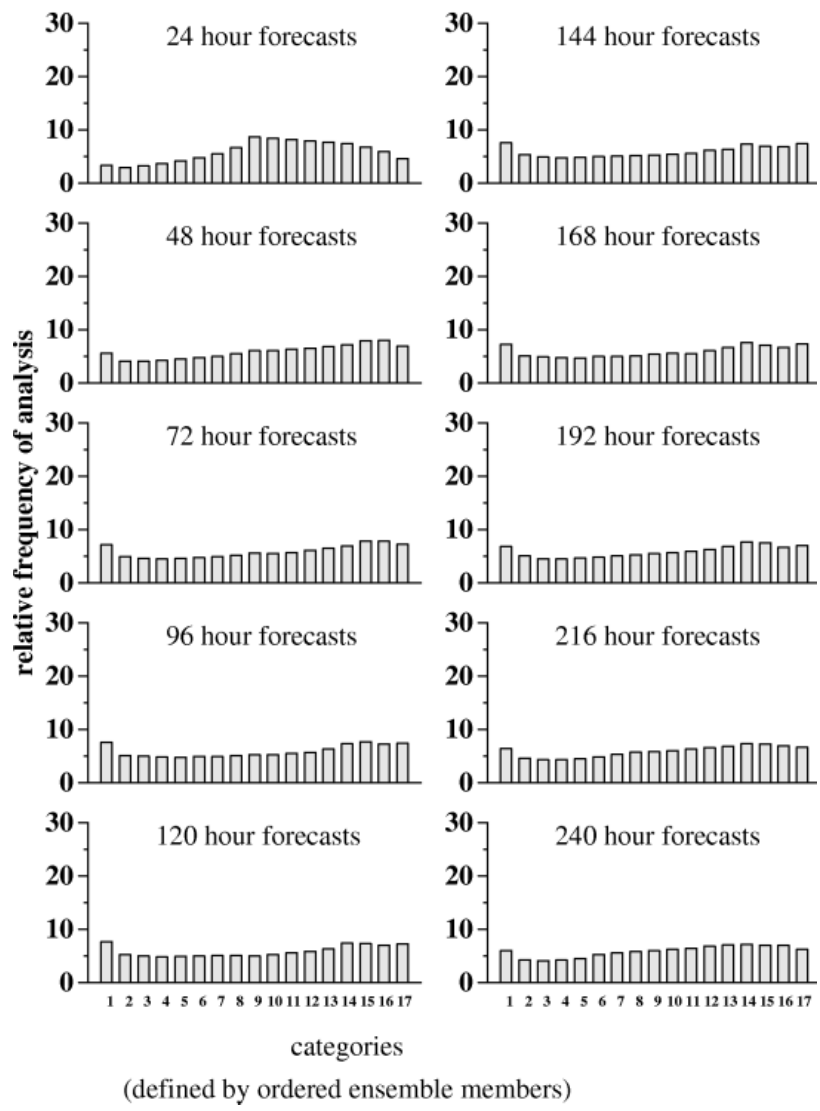

Fig. 3. Talagrand diagrams for height at $500 \mathrm{hPa}$ over global area for February 2001 for lead times from 24 to 240 h, for low resolution model $(192 \times 96)$.

\subsection{Relative Operating Characteristic curves for precipitation}

The Relative Operating Characteristic (ROC) curves have been proposed by Mason (1982) as a verification measure for ensemble forecasts. The method is based on ratios that measure the proportions of events and non-events for which warning can be provided. The ratios provide estimates of the probabilities that an event will be for-warned (hit-rate) and that an incorrect warning will be provided for a non-event (false-alarm). In a ROC curve, the hit rate is shown as a function of the false alarm rate. The surface under the curve gives a good indication of the performance of the system. It is worth noting that a ROC area of 0.5 is the no-skill level, with the arbitrary value of 0.7 being accepted as the lower limit of usefulness (see Mason, 1982). The detection of precipitation events (Hit rate) and non-events are obtained from a data set of nearly 160 stations distributed across Canada. These statistics are tabulated every 3 months and have been carried since early 1997 . Figure 5 shows the ROC curves for the low (a) and high (b) resolution ensemble sets. These re-
Talagrand diagrams for $500 \mathrm{hPa}$, global area February 2001
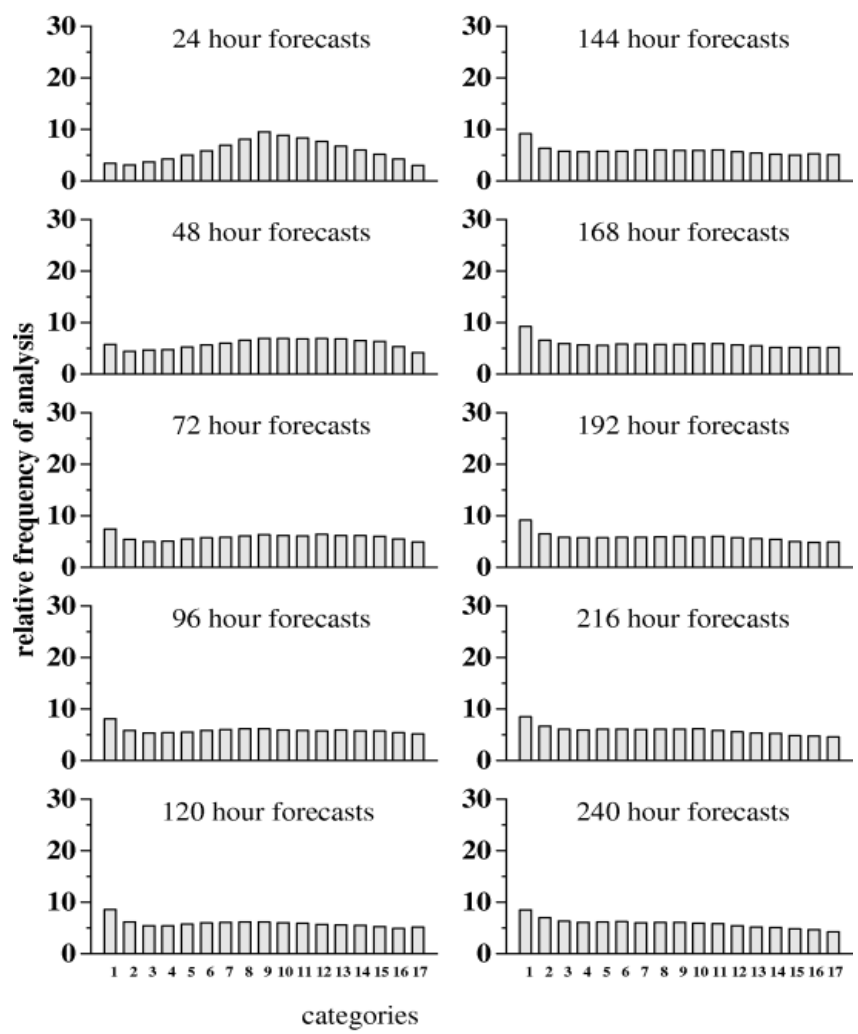

(defined by ordered ensemble members)

Fig. 4. Talagrand diagrams for height at $500 \mathrm{hPa}$ over global area for February 2001 for lead times from 24 to 240 h, for high resolution model $(300 \times 150)$.

sults are for the $5 \mathrm{~mm}$ threshold at 4 lead times (day 1, day 4, day 7 and day 10). They were calculated for the 3 month period starting in December 2000 and ending February 2001. The area under the curves (see Table 2) shows that both sets demonstrate skill up to 10 days and that the high resolution is better at all lead times.

Increasing the resolution to improve on the precipitation forecasts was also demonstrated with the ECMWF ensemble system (Buizza et al., 1998). Interestingly this relative gain is about the same as that obtained when the ensemble predictions system was increased in size from 8 to 16 members, which is shown under LOW(8) for 8 members. Generally speaking, Table 2 shows that an increase in ensemble resolution has a positive impact on the ensemble skill, due mostly to better topography related precipitation forecasts.

It is interesting to plot the variation of the areas as a function of the lead time. Figure 6 shows the plot of the low resolution scores (in dashed red) along with the high resolution scores (in solid black). As mentioned earlier, the improvement is apparent up to day 7, marginally so there after. After re-sampling our scores into shorter periods (10 days), 


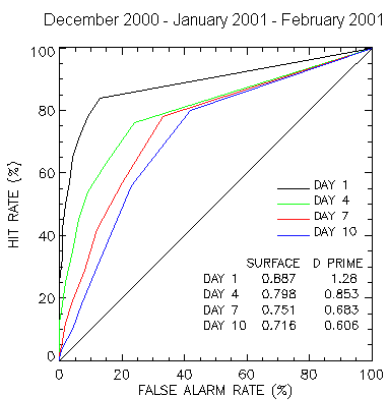

a) Low resolution verification ( $5 \mathrm{~mm}$ threshold)

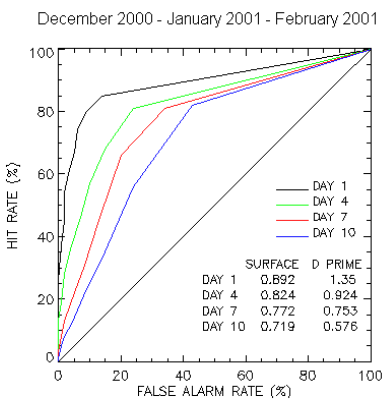

b) High resolution verification ( $5 \mathrm{~mm}$ threshold)

Fig. 5. Relative Operating Characteristic curves for $5 \mathrm{~mm}$ threshold for Winter 2000-01 (Dec-Jan-Feb) for low resolution models $(192 \times 96)$ in $(\mathbf{a})$ and high resolution models $(300 \times 150)$ in $(\mathbf{b})$.

Table 2. Comparison of ROC areas between high and low resolution (16 and 8 members)

\begin{tabular}{lccc}
\hline & high & low (16) & low (8) \\
\hline day 1 & 0.892 & 0.887 & 0.870 \\
day 4 & 0.824 & 0.798 & 0.781 \\
day 7 & 0.772 & 0.751 & 0.730 \\
day 10 & 0.719 & 0.716 & 0.683 \\
\hline
\end{tabular}

we find the null hypothesis using student-t confidence level is rejected at $65 \%$ at day $1,75 \%$ at day $4,85 \%$ at day 7 but accepted beyond day 8 . It is clear that both sets differ slightly at the beginning, due to the highly deterministic nature of short lead time. The higher resolution forecasts show an increase in skill up to day 7, afterward both sets move towards the climatological threshold. There is a fair amount of seasonal variations in these ROC scores and it is also interesting to plot the annual variations with time. Figure 7 shows four consecutive winter scores for the $5 \mathrm{~mm}$ threshold. The 4 consecutive years are; 1997-1998 in blue (8 members), 19981999 in grey (8 members), 1999-2000 in green (16 members) and 2000-2001 in red (16 members), all of which are computed from the common grid of $192 \times 96$ points. The comparison with the previous plots indicate that the annual variability is as great as the improvement obtained by the increase of resolution to $300 \times 150$ grid points.

The winter verifications (Figs. 6 and 7) show that forecasts have utility up to and even beyond day 7 . These conclusions are similar for the 2 and $10 \mathrm{~mm}$ thresholds, the $25 \mathrm{~mm}$ threshold is not verified because of the sample being too small.

\section{Future work}

The first priority is to make full use of the 16-member ensemble, extending the generation of our products in terms of probabilistic forecasts. Probabilities thus obtained will be used in the production of worded forecasts up to day 10 . Verification methods will also include the Probability Distribu-

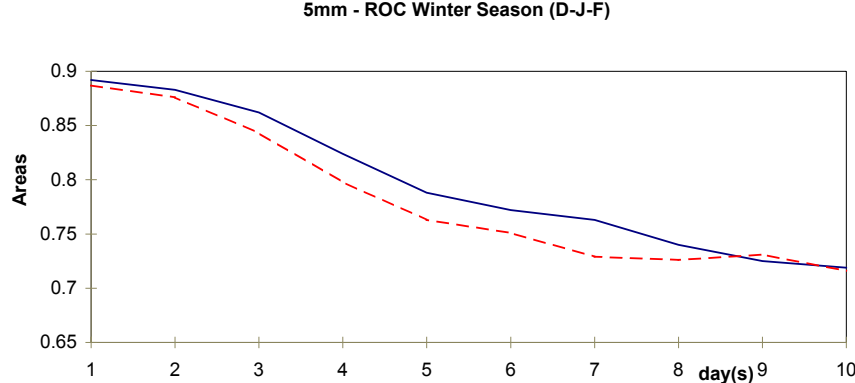

Fig. 6. Comparison of ROC curves for the $5 \mathrm{~mm}$ threshold for Winter 2000-01 (Dec-Jan-Feb) for high resolution models in solid black and low resolution models in dashed red.

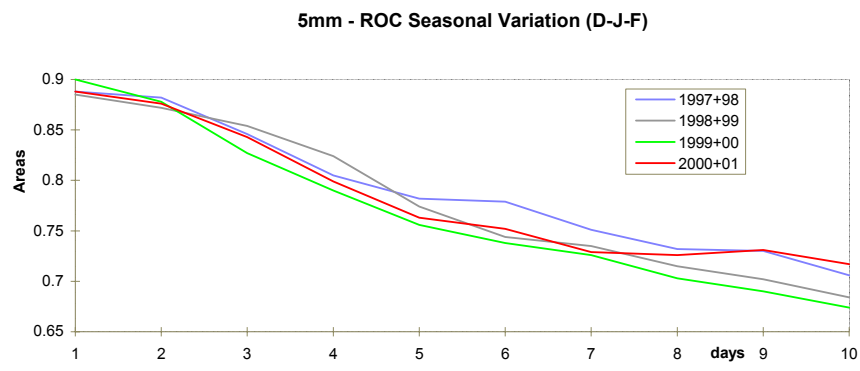

Fig. 7. Comparison of ROC curves at the $5 \mathrm{~mm}$ threshold for Winter season starting 1997-1998 in blue, 1998-1999 in grey, 1999-2000 in green and ending 2000-2001 in red. (All low resolution).

tion Function, as proposed by Wilson et al. (1999).

Second, at some point in the near future, the OI code will be replaced by an equivalent resolution ensemble Kalman filter. Since 1996, a great deal of effort has been concentrated on the development of an ensemble Kalman filter (see Mitchell and Houtekamer, 2000; Houtekamer and Mitchell, 2001; Mitchell et al., 2002). It is currently possible to assimilate a full set of observations with one of the latest version of the GEM model. The ability of the Kalman filter to provide the analyses for the medium-range ensemble prediction system is being examined.

Third, exchanges with NCEP have started in order to produce "grand ensemble" products. This collaboration is likely to be continued.

\section{References}

Buizza, R., Petroliagis, T., Palmer, T. N., Barkmeijer, J., Hamrud, M., Hollingsworth, A., Simmons, A., and Weddi, N.: Impact of model resolution and ensemble size on the performance of an ensemble prediction system, Q. J. R. Meteorol. Soc., 24, 19351960, 1998.

Côté, J., Gravel, S., Méthot, A., Patoine, A., Roch, M., and Staniforth, A.: The Operational CMC/MRB Global Environmental 
Multiscale (GEM) Model: Part I - Design Considerations and Formulation, Mon. Wea. Rev., 126, 1373-1395, 1998.

Dastoor, A. P.: Cloudiness parameterization and verification in a large-scale atmospheric model, Tellus, 46A, 615-634, 1994.

Houtekamer, P. L.: Global and local skill forecasts, Mon. Wea. Rev., 121, 1834-1846, 1993.

Houtekamer, P. L., Lefaivre, L., Derome, J., Ritchie, H., and Mitchell, H. L.: A system simulation approach to ensemble prediction, Mon. Wea. Rev., 124, 1225-1242, 1996.

Houtekamer, P. L. and Mitchell, H. L.: A Sequential Ensemble Kalman Filter for Atmospheric Data Assimilation, Mon. Wea. Rev, 129, 123-137, 2001.

Lefaivre, L., Houtekamer, P. L., Bergeron, A., and Verret, R.: The CMC Ensemble Prediction System. Proc. ECMWF 6th Workshop on Meteorological Operational Systems, Reading, U.K, ECMWF, 31-44, 1997.

Mason, I.: A model for the assessment of weather forecasts, Aust. Meteor. Mag., 30, 291-303, 1982.

McFarlane, N. A.: The effect of orographically excited gravity wave drag on the general circulation of the lower stratosphere and troposhere, J. Atmos. Sci., 44, 1775-1800, 1987.

McLandress, C. and McFarlane, N. A.: Interactions between orographic gravity wave drag and forced stationary planetary waves in the winter Northern Hemisphere middle atmosphere, J. Atmos. Sci., 50, 1966-1990, 1993.

Mitchell, H. L. and Houtekamer, P. L.: An Adaptive Ensemble Kalman Filter, Mon. Wea. Rev., 128, 416-433, 2000.

Mitchell, H. L., Houtekamer, P. L., and Pellerin, G.: Ensemble Size, Balance and Model-Error Representation in an Ensemble Kalman Filter, Mon. Wea. Rev. 130, 2791-2808, 2002.

Moorthi, S. and Suarez, M. J.: Relaxed Arakawa-Schubert: A parameterization of moist convection for general circulation models, Mon. Wea. Rev., 120, 978-1002, 1992.

Ritchie, H.: Application in the semi-Lagrangian method to a multilevel spectral primitive-equations model, Quart. J. Roy. Meteor. Soc., 117, 91-106, 1991.

Rutherford, I. D.: Data assimilation by statistical interpolation of forecast error fields, J. Atmos. Sci., 29, 809-815, 1972.

Talagrand, O., Vautard, R., and Strauss, B.: Evaluation of probabilistic prediction systems, Proceedings, ECMWF Workshop on Predictability, 1997.

Wagneur, N.: Une évaluation des schémas de type Kuo pour le paramétrage de la convection, Msc Thesis, UQAM, 76, 1991.

Wilson, L. J., Burroughs, W. R., and Lanzinger, A.: A Strategy for Verification of Weather Element Forecasts from an Ensemble Prediction System, Mon. Wea. Rev., 127, 956-970, 1999. 